\title{
XIV.
}

\section{Casuistische Mittheilungen aus der Poliklinik für Ohren- kranke zu Halle}

\author{
von \\ Dr. Adolf Eysell, \\ Assistenz-Arzt an der medicinischen Klinik.
}

\section{Fall.}

Caries des rechten Schläfenbeins. Thrombose des Sinus transversus. Phthisis Pulmonum. Amyloide Degeneration der Nieren. Tod.

Anna 0., 61/2 Jahr alt, aus Halle, von gesunden Eltern abstammend, erkrankte im vierten Lebensjahre an Keuchbusten, der Monate lang in grosser Heftigkeit anhielt. Intercurrent litt das Kind an Rachen-Diphtheritis.

Von dieser Affection genesen, klagte Patientin über heftige Schmerzen im rechten Ohre, welche mit dem Eintritt einer Otorrhöe beträchtlich nachliessen.

Nach einigen Wochen begann die Haut über dem Processus mastoideus dieser Seite sich zu röthen und war auf Druck sehr schmerzhaft; bald trat Schwellung hinzu, die grösser und grösser;wurde.

Im Juni 1871 liess der Tumor deutliche Fluctuation erkennen und entleerte anf ausgiebige Incision eine reichliche Menge stinkenden Eiters. Die eingeführte Sonde stiess auf in ziemlich grosser Ausdehnung entblössten Knochen und konnte gebogen durch eine in seiner hinteren Wand befindliche Fistelöffnung leicht in den äusseren Gehörgang gebracht werden.

Inzwischen hatte sich wohl das convulsivische Gepräge des Keuchhustens verloren, eine vollständige Genesung trat aber nicht ein; es blieb ein mässiger, zeitweise exacerbirender Bronchialkatarrh zurück. 
Nach Anlegung der Gegenöffnung hörten die Ohrschmerzen vollständig auf und besserte sich unter Beobachtung scrupulösester Reinhaltung der eiternden Hohlräume (mehrmals täglich vorgenommenes Durchleiten eines Stromes von, sehwacher Lösung von Kalihypermanganicum mittelst der Weber'schen Nasendouche) und Verordnung einer roborirenden Diät der Allgemeinzustand des Kindes in erfreulichster Weise.

Im Herbst 1871 traten die bronchitischen Erscheinungen wieder mehr in den Vordergrand und liessen sich im December desselben Jahres Herderkrankungen in deñ Lungen nachweisen. Gleichzeitig entstandeu von Neuem Schmerzen im rechten Ohre und steigerten sich bald zu heftigstem Allgemeinkopfschmerz, der nach mehrwöchentlichen Bestehen langsam wieder verschwand.

Mit dem Eintritt der besseren Jahreszeit hoben sich die Kräfte der Patientin etwas; die Eiterung aus der Fistelöffnung hinter dem rechten Ohre aber versiegte trotz aller angewandten Mittel nicht. Ende Juli 1872 wurde mittelst der Kornzange ein Sequester, grösser wie eine Bohne, aus der Eiterhöhle im Warzenfortsatze extrahirt. Jetzt schien der Process zur Ausheilung sich anschicken zu wollen, die Schmerzen kehrten nicht mehr wieder, der eitrige Ausfluss wurde äusserst spärlich.

Aber leider hatte inzwischen die Lungenaffection weitere Fortschritte gemacht. Die rauhere Jahreszeit fesselte Patientin ganz an das Zimmmer. Es trat hectisehes Fieber auf, welches rapiden Kräfteverfall im Gefolge hatte, bei geringem Eiweissgehalte des Urins gesellten sich Oedeme der Unterextremitäten, des Gesichts dazu, und das Kind erlag am 16. November v. J. seinem Leiden.

Section 20 h. p. m.

Leiche sehr abgemagert. Anasarka der unteren Extremitäten bis zu den Kniegelenken. Panniculus adiposus fast vollständig geschwunden. Musculatur schlaff.

Beim Eröffnen des Thorax sinken die Lungen wenig zurück, trotzdem liegt der Herzbentel viel grösser als normal zu Tage.

Herz hypertrophirt, namentlich der rechte Ventrikel, welcher bedeutend weiter und dickrandiger, als der linke erseheint. Klappenapparat normal. Durchmesser der Arteria pulmonalis 21/2 Mal so gross als der der Aorta.

Pleurablätter anf beiden Seiten, namentlich in ihren oberen Theilen, mit einander verwachsen.

Lungen beiderseits hochgradig verändert. In den Spitzen zahlreiche Verkalkungen, in den nächstfolgenden Partien käsige Infiltration im Zerfall begriffen (links oben eine grössere Caverne). Dann käsige Einlagerungen jüngeren Datums, darunter noch ziemlich frische und noch nicht verküste peribronchitische Herde und schliesslich Emphysem in den Basaltheilen beider Lungen.

Leber und Milz zeigen nichts Besonderes.

Glomeruli and kleinere Nierenarterien amyloid entartet.

Am Gehirn und seinen Hänten keine Veränderungen wahrunehmen. 
Im rechten Sinns transversus steckt mit der Gefüsswand untrennbar verbunden ein alter (wahrseheinlich im December 1871 entstandener) vollständig bindegewebig gewordener Thrombus, weleher vom Toreular Herophili bis beinahe znm Bubus der Vena jugularis reicht. Das Emissarium mastoidenm ist ebenfalls in einen Bindegewebsstrang verwandelt.

Linkes Gehörorgan wurde nicht nntersucht. Der Befund des aus dem Schädel entfernten rechten war folgender:

Dura mater von der oberen Fläche der Pyramide schwer abziehbar. In der Höhe des Meat. audit. intern. ist die Knochenwand des Sulcus transversus so dünn, dass die (durch Fänlniss) verfärbten Weichgebilde im Warzenfortsatze gral-grünlich durchschimmern. Nekrotische Knochenpartien nirgends vorhanden.

Aeusserer Gehörgang eng. Auskleidende Weichtheile verdickt. Seine hintere Wand wird von einer 3 Millim, im Durchmesser haltenden, ub berhäuteten Knochenfistel durchbrochen, deren Entfernung von der oberen Trommelfellinsertion 7 Millim. beträgt. Dieser Gang führt in eine einzige haselnussgrosse Caverne, welche die Stelle des Antrum und der nekrotisch ansgestossenen Scheidewände der Warzenzellen einnimmt und durch den zweiten die Ausssenwand des Processus mastoideus durchbohrenden Fistelgang direct mit der äusseren Atmosphäre communicirt. Eine dicke pyogene Membran überzieht die Ẅände dieser Höhle und lässt nirgends blossliegenden Knochen erkennen.

Das Tegmen tympani ist sehr stark verdickt und wie alle Theile der Pyramide im höchsten Grade sklerotisch.

Trommelfell und Perforationsnarbe eingezogen und in ihrer ganzen Ausdehnung dureh straffes Bindegewebe mit der Labyrinthwand verwachsen.

Hammergriff und Proc. brevis vollständig erhalten, Hammerkopf knöehern mit dem Tegmen verwachsen. Amboskörper und kurzer Fortsatz nichts Abnormes zeigend. An der Basis des llangen Fortsatzes, der bis zur Hälfte verloren gegangen ist, erheben sich drei relativ lange, scharf zugespitzte Osteophyten. Stapesköpfchen und angrenzende Schenkeltheile fehlen. Fussplatte im ovalen Fenster beweglich. Tuba durehgängig.

2. Fall.

Fractur der Sebädelbasis. Ruptur des Trommelfells und Blutung aus dem linken Ohre. Linksseitige Facialisparalyse. Heilung.

Friedrieh K., 24 Jahr alt, Landwirth aus Wieskan bei Löbejün, stürate am 4 . Oetober v. J. von einem Leiterwagen, welchen durchgehende Pferde in rasender Eile dahinführten. Besinnungslos wurde Patient nach Hause getragen, es floss Blut aus seinem linken Ohre. 
Drei Tage lag K. komatös da, dann kehrte das Bewusstsein zurück und nun besserte sich der Zustand des Kranken ausserordentlich rasch. Nach acht Tagen konnte Patient bereits das Bett verlassen. Die einzigen in die Augen fallenden Residnen der erlittenen Verletzung waren eine beträchtliche Abnahme des Hörvermögens und eine Facialislähmung anf der linken Seite.

Wegen letzterer Affection wurde Patient von seinem Arzte an die hiesige medicinische Klinik behufs Behandlung mit dem constanten Strom gewiesen und stellte sich am 16. October $1872 \mathrm{zum}$ ersten Male mir vor. Es waren damals Ptosis, Mundverziehnng und Schiefstand der Uvula vorhanden.

Die Uhr wurde linkerseits beim Andrücken gehört, rechts war die Hörweite normal. Stimmgabel vom Scheitel nach links gehört.

In den unteren hinteren Theilen des änsseren Gehörganges lagen eingetrocknete Blutkrusten. Das Trommelfell liess einen linienförmigen, horizontal verlaufenden Einriss mit verklebten Rändern erkennen, welcher das untere Ende des Hammergriffs fast tangirte. Die übrigen Theile der Membran waren leicht getrübt, die Hammergefässe injieirt.

$\mathrm{Da}$ K. versicherte, dass die functionelle Störung des linken Gehörorganes in den letzten Tagen schon wesentlich zurückgegangen sei, verhielt ich mich der Ohraffection gegenüber vollkommen exspectativ.

Drei Wochen später war die Rnpturnarbe noch deutlich zu sehen, Injection der Hammergefüsse verschwunden, Trommelfell von normaler Farbe. Hörweite für die Uhr beinahe 2 Fuss.

Unter consequenter, täglich vorgenommener Anwendung des constanten Stromes gingen, wenn auch langsam, die Symptome der Facialisparalyse zuriek und Patient konnte am 14. Jan. d. J. fast vollständig geheilt entlassen werden.

\section{Fall.}

Phthisis pulmonum. Defect des rechten Trommelfelles. Partielle Nekrose der Gehörknöchelchen. Sectionsbefund.

Benno T. 33 Jahr alt, Kellner, ans Halle, bekam am Pfingstfeste v. J., während er sich sonst ganz gesund fühlte und früher nie an der Brust gelitten zu haben behauptete, einen Blutsturz. Zehn Tage musste er das Bett hüten und fühlte sich danach so schwach, dass er seinen Geschäften nicht wieder nachgehen konnte.

Am 5. October v. J. kam T. in die hiesige otiatrische Poliklinik mit Klagen über heftiges Sausen und Schmerzen im rechter Ohre und in der Schläfengegend derselben Seite.

Patient zeigte einen exquisit phthisichen Habitns; im rechten oberen Lappen waren schon damals Cavernen nachzuweisen. 
Die Ohrinspection ergab das Vorhandensein eines rechtsseitigen subacuten Katarrhs der Paukenschleimhaut. Trommelfell trübe. Starke Injection der Hammergefässe. Radiäre venöse Streifung. Uhr beim Anlegen, Stimmgahel von allen Theilen des Kopfes nach rechts gehört.

Therapie: 2 Blutegel vor das rechte Ohr.

12. Oct. 72. Die Schmerzen im Ohre haben nachgelassen. Schläfenkopfschmerz und das höchst quälende Sausen bestehen fort.

Gegen letzteres wurde von Prof. Sehwartze die Trommelfellparacentese gemacht.

15. Oct. 72. Otorrhöe eingetreten. Subjective Geränsche beinahe vollständig verschwunden. Hörweite unverändert.

Die künstliche Trommelfellperforation vergrösserte sich in der folgenden Zeit sehr rasch, so dass am

25. Nov. 72 zwei Drittel der Membran verlorep gegangen waren. Der Hammergriff ragte in der Länge von 2 Millimetern von Weichtheilen vollständig entblösst, intensiv weiss aussehend, in den Trommelfelldefect hinein. Paukenschleimhaut war geröthet und gewulstet.

Die chronisch-pneumonischen Processe hatten inzwischen immer grössere Partien des Lungengewebes ergriffen. Patient musste die meiste Zeit des Tages im Bette zubringen und wurde am 2. Dec. v. J. in die hiesige Medicinische Klinik aufgenommen.

Am 22. Januar d. J. war das rechte Trommelfell bis auf einen kleinen Rest um den Proc. brevis des Hammers verloren gegangen, vom Hammergriff nichts mehr wahrzunehmen. Die Eiterung hatte etwas nachgelassen.

In dieser ganzen Zeit beschränkte sich die Therapie auf Reinhalten der befallenen Theile durch lane Wassereinspritzungen.

Am 5. Febr. 73 war die Otomböe vollständig versehwunden und die Paukenschleimhaut von ziemlich normalem Aussehen.

Der Kräfteverfall des Kranken nahm stets zu, und am 12. Febr. a. c. erfolgte der Tod.

Section am 13. Febr. 73.21 h. p. m.

Cavernen in beiden Lungenspitzen. Fettleber. Keine Darmgeschwüre. Nirgends miliare Knötchen.

Section des rechten Ohres.

Aeusserer Gehörgang zeigt nichts Abnormes. Trommelfell ganz fehlend. Hammerkopf und Hammerhals sind vollständig erhalten und nicht nekrotisch. Langer Ambosschenkel verloren gegangen. An der Ursprungsstelle des letzteren ist der Amboskörper nekrotisch. Hammer-Ambosgelenk gesund. Sehne des Tensor tympani zerstört, Muskelsubstanz in fettiger Degeneration begriffen, das Rostrum cochleare nekrotisch. Stapes erhalten und beweglich, Köpfehen nekrotisch, Stapediussehne zu Grunde gegangen, Fensternische verengert. Paukenschleimhaut blass, etwas verdickt. Antrum und Cellulae mastoideae mässig gross und von eingedicktem Eiter erfüllt. 
Dura mater über den unteren Theilen der Schläfenschuppe und der oberen Pyramidenfläche etwas verdickt, auf diesen selbst sind zahlreiehe plattenförmige Auflagerungen von neugebildetem Knochen zu erkennen. Tegmen tympani verdickt und aus poröser Knochensubstanz bestehend. Sinus transversus nichts Abnormes zeigend.

\section{Fall.}

Zahlreiche Schleimpolypen im Antrum und den Cellulae mastoideae.

Hermann K., 4 Jahr alt, aus Halle, erkrankte am 20. Febr. d. J. an Rachendiphtheritis. Hinzugetretene croupöse Entzündung des Kehlkopfes machte die Tracheotomie nothwendig. Patient überlebte die Operation nur einen Tag.

Die am 26. Febr. 16 h. p. m. behufs Untersuchung des Verhaltens der Warzenzellen im kindlichen Alter vorgenommene Ohrsection ergab zufallig folgenden interessanten Befund:

Trommelfell rechts aufs Aeusserste eingezogen.

Alle Hohlräume des Mittelohres dieser Seite vollständig luftleer, mit zähem, schwach getrubbtem Schleime gefüllt.

Tuba durch Schleimhautschwellung verengt and durch zähes Secret gegen das Cavum pharyngo-nasale abgeschlossen.

Paukenschleimhant sehr beträchtlich verdickt, blutreich und gewalstet.

Die Schleimhant des Antrum und der wohl entwickelten Cellulae mastoideae zeigte ein gleiches Verhalten; aus ihr heraus hoben sich bis $2^{1 / 2}$ Millim. lange ausserordentlich dünne Stiele, welche intensiv rothe, meist kugelige Gebilde in der Grösse eines Stecknadelkopfes bis zu der eines Hanfkorns trugen.

Solcher Polypen wurden im Antrum 6, in den Warzenzellen 11 gezählt.

Die Stiele, welche so fein waren, dass man sie nur mit der grössten Mühe bei Entfernung der umgebenden Schleimmassen vor dem Zerreissen schützen konnte, bestanden aus einer bindegewebigen Grundsubstanz, in welcher zu- und abführende. Gefässe (bei den kleinsten nur je ein solches) verliefen. Im Polypenkörper waren diese Blutleiter dureh ein Netz sehr zierlicher Capillarschlingen verbunden, die durch ihre Anordnung an die Gefässvertheilung in dem Granulationsknöpfchen erimnerten. Cylinderepithel bedeckte diese Gebilde.

Linkerseits war die Mittelohrschleimhaut von durchaus normaler Beschaffenheit und die Tuba leicht durchgängig. 


\section{Fall.}

Doppelseitiger acuter Mittolobrkataru. Reohts interlamelläre Abscesse im Trommelfelle. Heilung.

Ernst W., 5 Jahr alt, aus Halle, bekam nach einer katarrhalischen Angina Schmerzen in beiden Ohren. Das Kind schlief sehr schlecht, schrie häufig im Schlafe anf und wurde schwerhörig.

Am 14. December v. J, dem dritten Tage nach dem Eintreten der Ohrenschmerzen, kam Patient in Behandlung.

Beide Trommelfelle waren vorgewölbt and von violetrother Farbe, einzelne Gefässe bei der Intensität der Tinction nicht zu unterscheiden.

Rechts (cf. beigedruckte Fig.) unmittelbar hinter dem Umbo war eine kreisförmige, flachgewölbte Erhabenheit von gelber Farbe

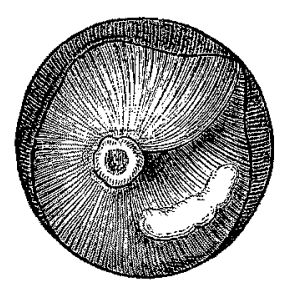
und mattem, wachsartigem Glanze zu sehen.

Eine ebensolche von bohnenförmiger Gestalt lag dem vorderen unteren Quadranten auf. Sie ging mit ihrem oberen, leicht concaven Rande unmerklich in die Trommelfelloberfläche über, die seitlichen und der untere Rand dagegen waren wallartig über das Niveau der Membranna tympani erhoben. Hammergriff durch die vorgebuchteten uberliegenden Theile

des Trommelfells verdeckt.

Therapie: Ein Blutegel vor jedes Ohr. Cort. frangul. zum Thee.

Auf Druck mit dem Sondenknopfe entstand in der kreisförmigen, am Umbo gelegenen Erhabenheit eine Delle (cf. Fig.), welehe am

15. Dee. noch vollkommen unverändert gesehen wurde. Das Trommelfellbild hatte sich auch sonst in nichts geändert. Der Schlaf des Patienten war in der vergangenen Nacht besser gewesen.

16. Dec. Röthe des Trommelfelles, wahrscheinlich in Folge von seröser Durchfeuchtung der Epidermis, weniger intensiv. Delle noch deutlich zn erkennen.

In der Nacht vom 16. auf den 17. hat sich unter erheblichem Nachlass der Schmerzen rechts Otorrhöe eingestellt.

17. Dec. Im Sinus meat. andit. extern. liegt wenig Eiter, welcher nach rom und oben ein dentlich pulsirendes Lichtbildchen zeigt.

Das Trommelfell hat in Ganzen seine Form bewahrt, sieht feucht und gleichmässig graul-weiss ans; die gelben, umschriebenen Erhabenheiten sind vollständig verschwunden.

Beim Ausspritzen des Ohres mit lauem Wasser wird wenig dünner Eiter und Epidermisfetzen entleert.

Die fein granulirt anssehende, intensiv rothe Cutisschicht des 
Trommelfells liegt entblösst zu Tage und lässt die radiär verlaufenden stark injicirten Gefässe in schönster Weise erkemen.

Perforation $\mathrm{nich}$ t vorlanden.

Schmerzen kamen nicht wieder.

Der Ausfuss versiegte bald vollständig nnd das mehr erblassende Trommelfell bedeckte sich mit einer nenen Epidermisschicht.

Unter Anwendung des Polizerschen Verfahrens wurde der Mittelohrkatarrh auf beiden Seiten gehoben und Patient konnte am 20. Januar d. J. geheilt entlassen werden. 